\title{
EFFECTS OF PRESS PRESSURE ON THE MECHANICAL PROPERTIES OF RIB ROOT FOR GLASS FIBER REINFORCED POLYPROPYLENE COMPOSITES, MOLDED BY PRESS AND INJECTION HYBRID MOLDING
}

\author{
KAZUTO TANAKA, KAITO KARASUNO, NOGUCHI RYUICHI \& TEUTA KATAYAMA \\ Department of Biomedical Engineering, Doshisha University, Japan
}

\begin{abstract}
The press and injection hybrid molding system, which is a novel molding technology with the combination of press molding and injection molding, is expected for the production of FRTP (Fiber Reinforced Thermoplastics) with complicated shapes and high levels of stiffness and strength. Press and injection hybrid molded structures consist of an outer shell laminate of continuous fiber and injected short or long fiber reinforced thermoplastics which form the rib structure. The higher mold temperature was reported to increase the strength of the interface between the outer shell laminate and injected material. On the other hand, the penetration of continuous fibers of the outer shell laminate into the rib structure decreases the mechanical properties of the outer shell laminate. While the effects of mold temperature on the mechanical properties of hybrid molded composites were clarified, the effects of press pressure have not been clarified yet. In this study, the effects of the press pressure on the mechanical properties of the outer shell laminate and interfacial strength between the outer shell laminate and injected material were evaluated. As the lower press pressure decreases, the penetrated height of continuous fiber into the rib structure, higher in-plane tensile strength and lower interfacial strength, are obtained.
\end{abstract}

Keywords: GFRP, injection molding, press molding, press and injection hybrid molding, preheating, rib root, interfacial strength.

\section{INTRODUCTION}

The use of fiber reinforced thermoplastic composite material (FRTP) is expected to contribute to weight reduction of automobiles because it has high specific strength and high specific rigidity, and can be recycled [1], [2]. Since carbon fiber-reinforced thermoplastic composites (CFRTP) are expensive [3], their application to mass-produced automobiles is limited. On the other hand, the application of glass fiber-reinforced thermoplastic composites (GFRTP) is expected due to its affordable price.

While traditional injection molding used for GFRTP can mold complex shapes, it is not suitable for the production of structural parts that require high mechanical properties because of the limitation of the fiber length [4]. Although continuous fiber reinforced thermoplastic composites has excellent mechanical properties, it is difficult to mold complex shaped products such as the ribs and bosses because of the limitation in their formability [5], [6].

Recently, to mold FRTP with excellent strength and stiffness into complex shaped products with ribs and bosses, the hybrid molding process has been developed by combining press molding and injection molding [7]. In hybrid molding it was reported that the products fracture at the interface between the outer shell laminate and the rib [8]-[10], so it is necessary to increase the interfacial strength between the outer shell laminate and the rib. The higher interfacial strength was reported to be obtained by raising the temperature of preheating of the materials or mold temperature which can keep the temperature of the outer shell laminate above the melting point of the matrix resin. Although this hybrid molded structure has the higher interfacial strength, its continuous fiber in the outer shell laminate was disordered and 
these disordered fibers decreased the mechanical properties of the outer shell laminate. It is important to reduce the disorder of continuous fibers in the outer shell laminate by molding with a low press pressure.

In this study, glass fiber-reinforced polypropylene composites were molded by press and injection hybrid molding system at different press pressures. The in-plane tensile tests of outer shell laminates and the tensile tests using T-shaped specimens cut out from the molded products were performed to evaluate the in-plane tensile strength and the interfacial strength between the outer shell laminate and injected material.

\section{MATERIALS AND EXPERIMENTAL METHODS}

\subsection{Materials}

GF/PP prepreg sheets (NEOMATEX, KURABO Industries Ltd, $\mathrm{V}_{\mathrm{f}}=35 \%$, Thickness $=$ $0.5 \mathrm{~mm}$, melting point $=154^{\circ} \mathrm{C}$ ) that consist of continuous glass fiber twill woven fabrics and polypropylene (PP) as matrix were used for the thermoplastic composite laminates. Four prepreg sheets were stacked and press-molded at $210^{\circ} \mathrm{C}$ of molding temperature, $3 \mathrm{MPa}$ of pressure, and 360s of holding time to be used for the outer shell laminate of the press and injection hybrid molding. The GF/PP pellet (HG30U, Japan Polypropylene Co. Ltd) was used for the injection molding material.

\subsection{Press and injection hybrid molding}

The schematic drawing of the press and injection hybrid molding system (SATHO MACHINERY WORKS Co, Ltd) is shown in Fig. 1 and the molding condition, press pressure and maximum injection volume, is shown in Table 1. After the outer shell laminate was heated in advance using the heater (LEIBROCK, TH-5) at $245^{\circ} \mathrm{C}$, it was placed inside the mold held at $85^{\circ} \mathrm{C}$, and press-molded at $3 \mathrm{MPa}, 5 \mathrm{MPa}$ or $7 \mathrm{MPa}$. After press molding, $\mathrm{GF} / \mathrm{PP}$ was injected at $210^{\circ} \mathrm{C}$ on the outer shell laminate and the hybrid molded products shown in Fig. 2 was obtained. The black part is the outer shell laminate and the white part is the injected material. The specimens pressed at $3 \mathrm{MPa}, 5 \mathrm{MPa}$ and $7 \mathrm{MPa}$ are hereinafter referred to as h-3, h-5 and h-7, respectively, as shown in Table 1.

To understand the penetration behavior of the outer shell laminate into the rib, the hybrid molding products without injection (press only) were also molded at $3 \mathrm{MPa}, 5 \mathrm{MPa}$ and $7 \mathrm{MPa}$ (referred to as p-3, p-5 and p-7). Fig. 3 shows the typical hybrid molded products without injection (p-3).

\subsection{Mechanical testing}

In order to evaluate the in-plane tensile strength, in-plane tensile tests were conducted with a cross head speed of $0.017 \mathrm{~mm} / \mathrm{sec}$ at room temperature with a universal material testing machine (Auto graph, Shimazu) as shown in Fig. 4. In-plane specimens were cut out from the molded products and aluminum tabs were bonded to the both ends of the specimen using epoxy adhesive. In this study, the in-plane tensile strength was calculated by dividing the maximum fracture loads during the in-plane tensile tests by the cross-sectional area at the rib root.

In order to evaluate the interfacial strength between the outer shell laminate and the rib, T-shaped tensile tests were conducted with a cross head speed of $0.017 \mathrm{~mm} / \mathrm{sec}$ at room temperature with a universal material testing machine (5566, Instron) as shown in Fig. 5. $\mathrm{T}$-shaped specimens were cut out from the molded products and aluminum tabs were bonded 
using epoxy adhesive. In this study, the interfacial strength was calculated by dividing the maximum fracture loads during the T-shaped tensile tests by the cross-section area at the fracture. To evaluate the penetration behavior of the outer shell laminate into the rib root, the penetration height was measured for the hybrid molded specimens and the hybrid molded products without injection (press only). As shown in Fig. 6, the average height at the center of the rib measured at both ends of the specimen was defined as the penetration height.

A non-contact 3D deformation analyzed system (ARAMIS, GOMmbH) was used for the strain measurement of the T-shaped test specimens. A digital microscope (VHX-5000, KEYENCE) was used for the observation of the cross section of the specimens.

Table 1: Molding condition for press and injection hybrid molding.

\begin{tabular}{|l|l|l|}
\hline & Press pressure $[\mathrm{MPa}]$ & Injection volume $\left[\mathrm{m}^{3}\right]$ \\
\hline h-3 & 3 & $1.19 \times 10^{-4}$ \\
\hline h-5 & 5 & $1.16 \times 10^{-4}$ \\
\hline h-7 & 7 & $1.13 \times 10^{-4}$ \\
\hline
\end{tabular}

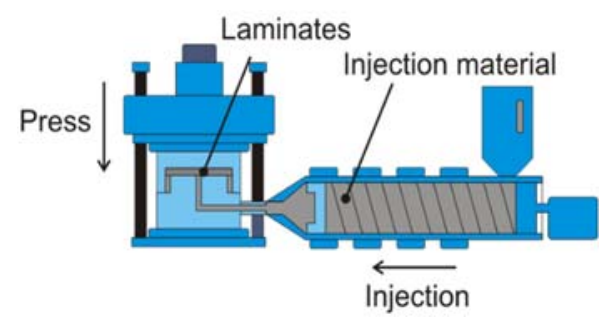

Figure 1: Schematic drawing of the press and injection hybrid molding process.

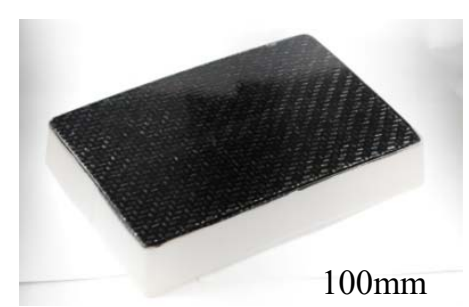

(a) Laminate side (press)

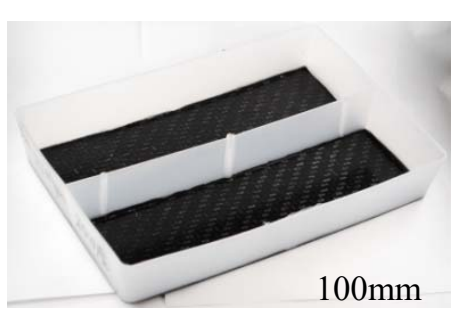

(b) Rib side (injection)

Figure 2: Molded product manufactured by press and injection hybrid molding.

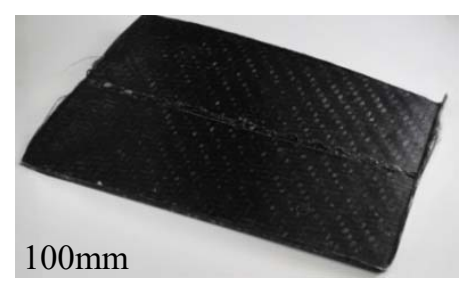

Figure 3: Schematic drawing of hybrid molded product without injection material (p-3). 


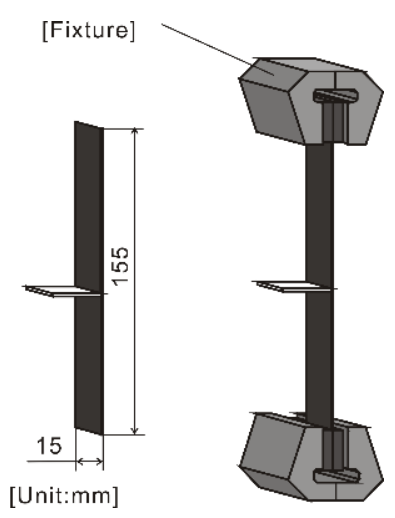

Figure 4: Schematic drawing of in-plane specimen and its tensile test.

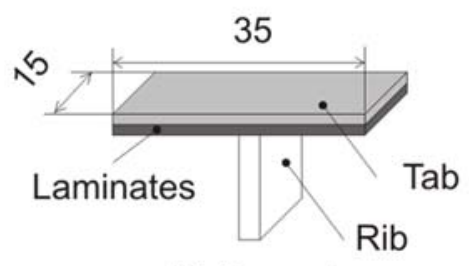

[Unit : $\mathrm{mm}$ ]

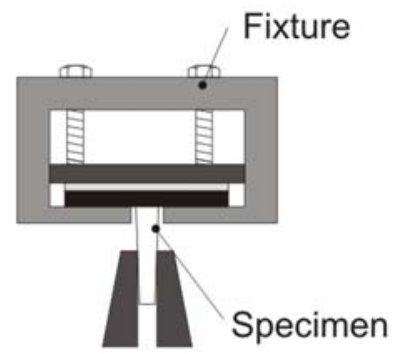

Figure 5: Schematic drawing of T-shaped specimen and its tensile test.

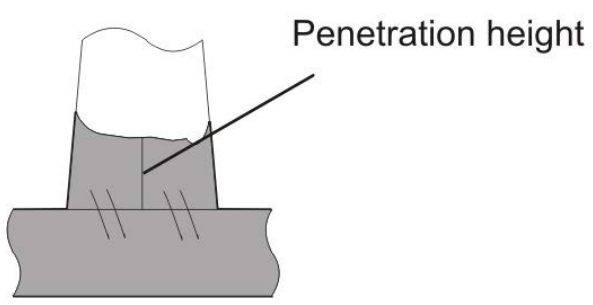

Figure 6: Measured penetration height of specimen.

\section{RESULTS AND DISCUSSION}

\subsection{Observation of cross section}

The cross-section views of the hybrid molded products without injection (p-3, p-5 and p-7) are shown in Fig. 7 and the penetration height into the rib root is shown in Fig. 8. After press molding, the continuous fiber and the resin of the outer shell laminate penetrated the rib depth direction. The central part (core) in the rib flow more than the skin and the shape of the outer shell laminate in the rib was convex. The higher press pressure increases the penetration height into the rib. 
The cross-section views of the hybrid molded specimens (h-3, h-5 and h-7) are shown in Fig 9 and the penetration height into the rib root is shown in Fig. 10. The shape of interface between the outer shell laminate and injected materials was concave and the higher press pressure increases the penetration height into the rib. Comparing the hybrid molding products without injection (press only) and the hybrid molded products at the same pressure, the penetration height of the hybrid product is lower than that of the hybrid molded products without injection (press only). It means that the interface moved to the rib root direction by injection molding. In injection molding, a method of forming a V-shaped interface by reflowing the resin near the weld has been developed [13]. In the hybrid molded products, the outer shell laminate was pushed back toward the rib root by injection molding after press molding and a U-shaped interface was formed. As the materials of the outer shell laminate close to the mold of the rib was cooled due to the cold mold, it remained at the both sides of the rib without being pushed back.

\subsection{In-plane tensile strength and interfacial strength}

The test results of the in-plane tensile tests are shown in Fig. 11. Ref in Fig. 11 shows the tensile strength of the outer shell laminate. As the press pressure became lower, the inplane tensile strength became higher, and the tensile strength of h-3 was almost same as Ref. The strain distribution of each specimen just before crack initiation is shown in Fig. 12. For h-3, the strain concentrated at the corner of the rib root. For h-5 and h-7, strain concentrated at the disorder part of the outer shell laminate. The side view of the specimen at the crack initiation and the fracture during in-plane tests are shown in Fig. 13. For h-3, the crack initiated at the corner of the rib root. For h-5 and h-7, crack initiated at the disorder part of the continuous fiber of the outer shell laminate that flowed into rib root by the press molding

The results of T-shaped tensile tests are shown in Fig. 14. Although the interfacial strength at the interface of p-7 was higher than that of p-3, there was no significant difference between other pair. Fig. 15 shows the strain distribution just before the crack initiation of the specimens during the T-shaped tensile test. For h-3 and h-5, the strain concentrated at the corner of the rib root. On the other hand, for h-7, the strain was concentrated at the corner of the rib root and the interface between the outer shell laminate and the injected material. The observation results of the T-shaped tensile test specimens at the crack initiation and the fracture are shown in Fig. 16. For h-3 and h-5, the crack initiated at the corner of the rib root and fractured along the interface. On the other hand, for h-7, cracks occurred at the corner of the rib root and at the interface end of the outer shell laminate and the injection material, the crack propagated along the continuous fiber layer of the outer shell laminate, and then broke at the interface.

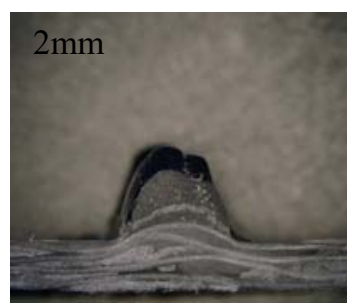

(a) p-3

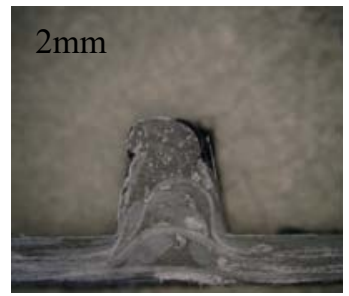

(b) p-5

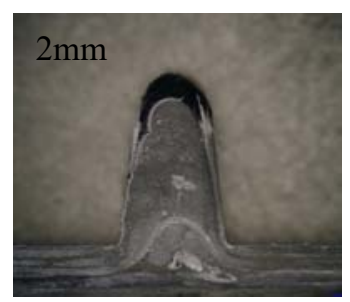

(c) p-7.

Figure 7: Magnified cross-section view of specimens for press molded products. 


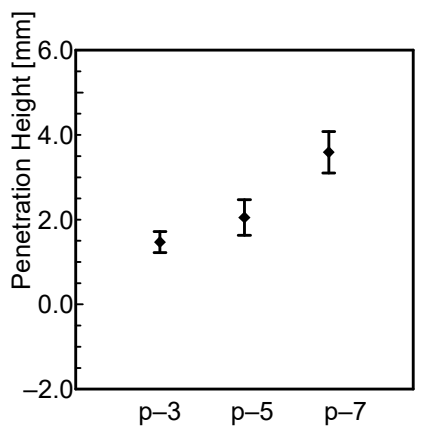

Figure 8: Penetration height of specimens for press molded products $(\mathrm{N}=5$, mean $\pm \mathrm{S}$. D.).

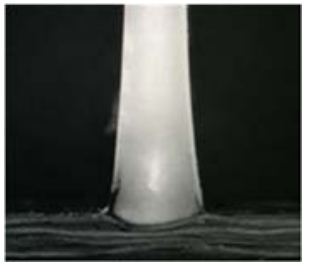

(a) h-3

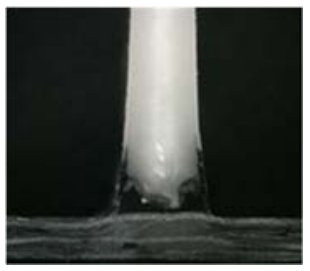

(b) h-5

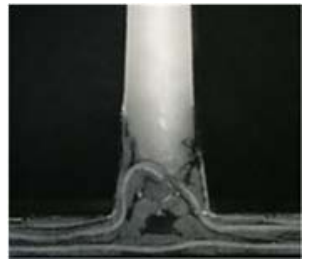

(c) h-7

Figure 9: Magnified cross-section view of specimens for hybrid molded products.

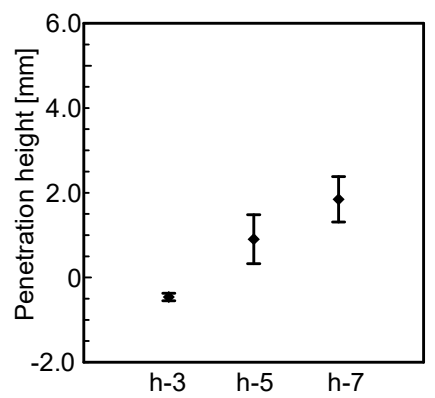

Figure 10: Penetration height of specimens for hybrid molded products $(\mathrm{N}=5$, mean \pm S. D.).

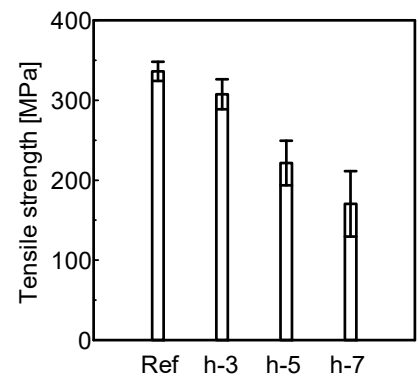

Figure 11: In-plane tensile strength $(\mathrm{N}=5$, mean $\pm \mathrm{S}$. D. $)$. 


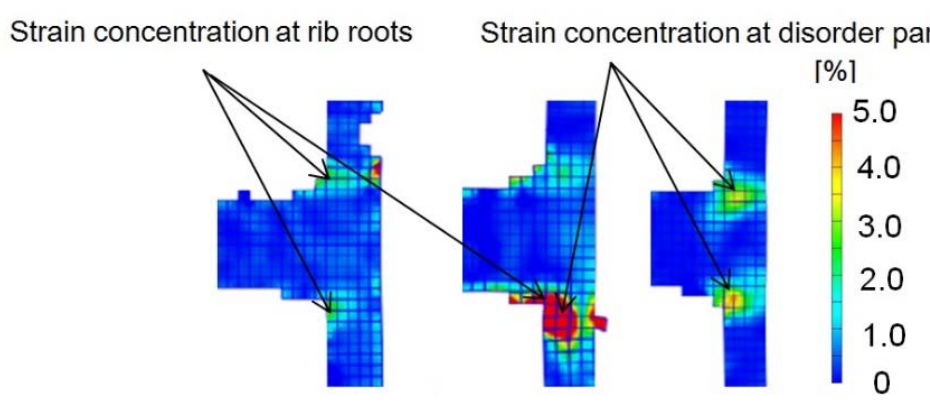

Figure 12: Strain distribution of in-plane specimens.

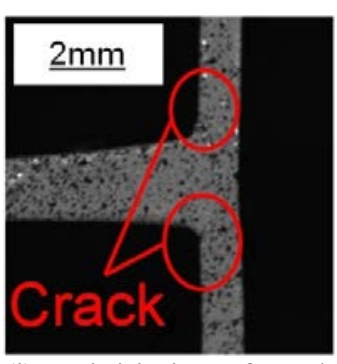

(i) At initiation of crack

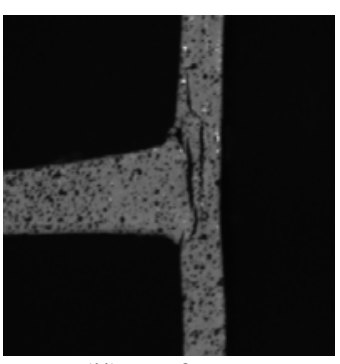

(ii) At fracture

(a) h-3

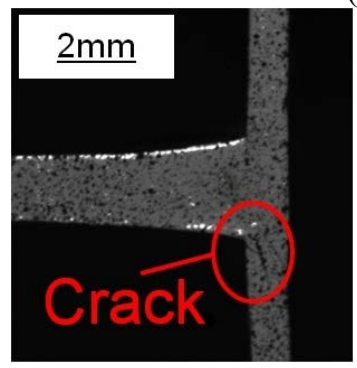

(i) At initiation of crack

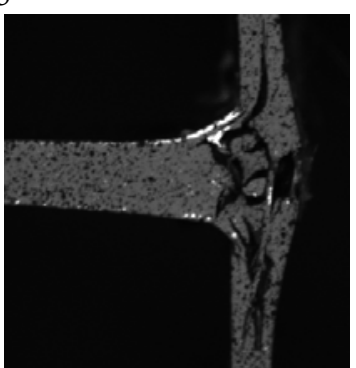

(ii) At fracture

(b) h-5

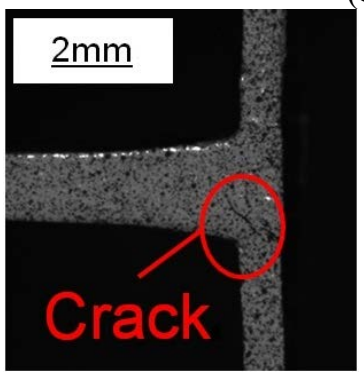

(i) At initiation of crack

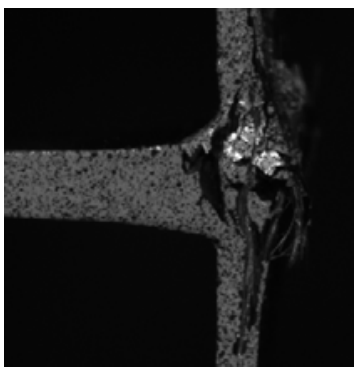

(ii) At fracture

(c) h-7

Figure 13: Side view of specimens during in-plane test. 


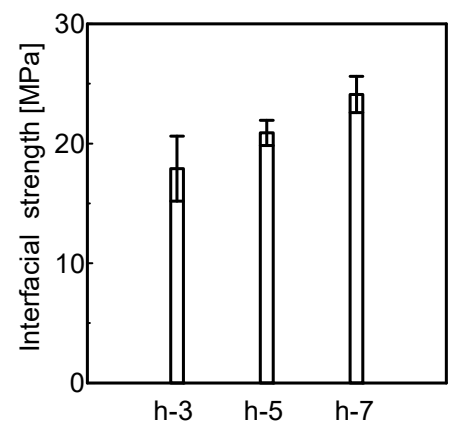

Figure 14: $T$-shaped tensile strength $(\mathrm{N}=5$, mean $\pm \mathrm{S}$. D.).

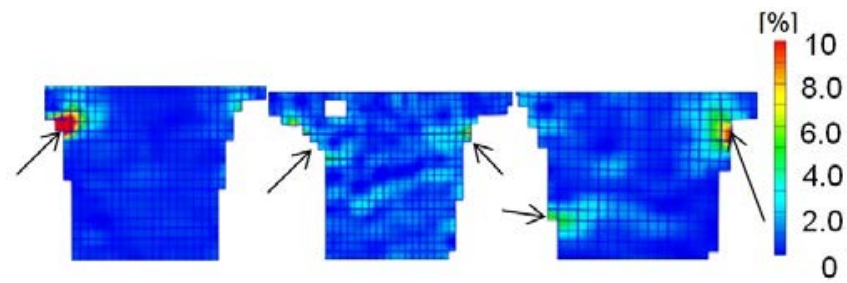
(a) h-3 ( $\varepsilon=0.5 \%)$
(b) h-5 ( $\varepsilon=0.5 \%)$
(c) h-7 $(\varepsilon=0.5 \%)$

Figure 15: Strain distribution of T-shaped specimen.

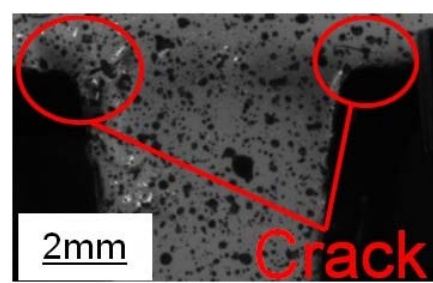

(a) h-3 - (i) At initiation of crack

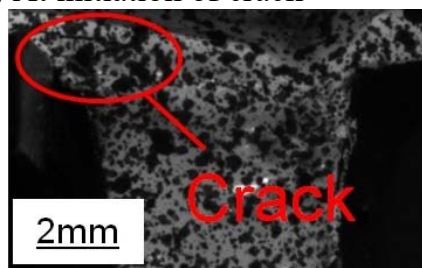

(b) h-5 - (i) At initiation of crack

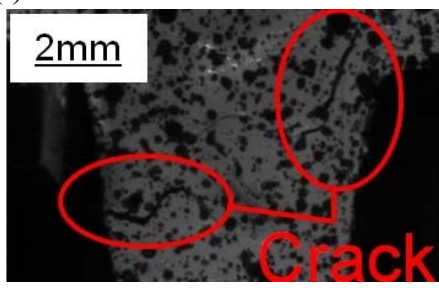

(c)h-7 - (i) At initiation of crack

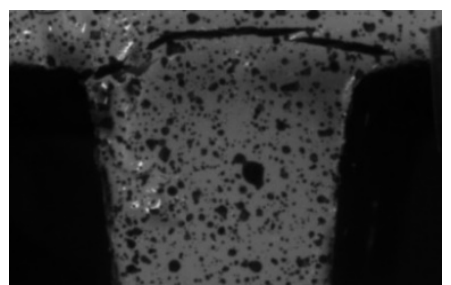

(ii) At fracture

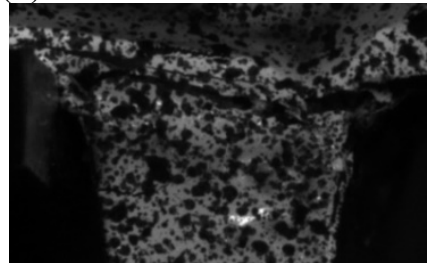

(ii) At fracture

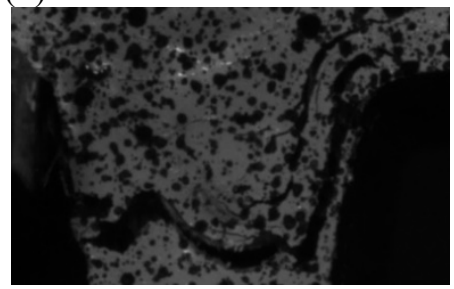

(ii) At fracture

Figure 16: Observation of specimens during T-shaped tensile test. 
In the case of h-3 having a low press pressure, the penetration height of the outer shell laminate into the rib at the press molding was low and penetrated outer shell laminate was pushed back toward the rib root direction by the injection material, and the disorder of the continuous fiber of the outer shell laminate became smaller. Therefore, higher in-plane tensile strength was obtained.

\section{CONCLUSION}

In this study, glass fiber-reinforced polypropylene composites were molded by press and injection hybrid molding system at different press pressures. The in-plane tensile tests of outer shell laminates and the tensile tests using T-shaped specimens cut out from the molded products were performed to evaluate the in-plane tensile strength and the interfacial strength between the outer shell laminate and injected material. The investigation yielded the following conclusions:

1. In the hybrid molded products, the outer shell laminate was pushed back toward the rib root by injection molding after press molding and a U-shaped interface was formed.

2. In the case of the specimen having a low press pressure, the penetration height of the outer shell laminate into the rib at the press molding was low and the disturbance of the continuous fiber of the outer shell laminate became smaller. Therefore, higher in-plane tensile strength was obtained.

\section{REFERENCES}

[1] Jacob, A., Car Makers Increase Their Use of Composites. Reinforced Plastics, 48(2), pp. 26-32, 2004.

[2] Frederique, M., Composites Penetration Growth in Automotive: Towards Mass Production 2010-2020 Trends and Forecasts. JEC Composites, pp. 13-22, 2011.

[3] Michael, F.A., Material selection in mechanical design, Butterworth-Heinemann, pp. 482-483, 2011.

[4] Morita, K., Tanaka, T., Imaida, Y. \& Inoue, A., Study on Injection Molding of Glass Fiber Reinforced Polypropylene with Fiber Length and Dispersion. Proceeding of the 86th JSME annual meeting, (114-1), 2011.

[5] Cabrera, N.O., Reynolds, C.T., Alcock, B. \& Peijs, T., Non-Isothermal Stamp Forming of Continuous Tape Reinforced All-Polypropylene Composite Sheet. Composites Part A: Applied Science and Manufacturing, 39(9), pp. 1455-1466, 2008.

[6] Zhu, B., Yu, T.X., Zhang, H. \& Tao, X.M., Experimental Investigation of Formability of Woven Textile Composite Preform in Stamping Operation. Composites Part B: Engineering, 42(2), pp. 289-295, 2011.

[7] Harte, A.M., \& McNamara, J.F., Use of Micromechanical Modelling in the Material Characterization of Overinjected Thermoplastic Composites. Journal of Materials Processing Technology, 173(3), pp. 376-383, 2006.

[8] Fiorotto, M. \& Lucchetta, G., Experimental Investigation of a New Hybrid Molding Process to Manufacture High-Performance Composites. International Journal of Material Forming, 6(1), pp. 179-185, 2013.

[9] Asanuma, N., Applying Direct Injection Molding Process and Hybrid Molding System to CFRP Molding. Journal of JSPP, 27(3), pp. 89-93, 2015.

[10] Tanaka, K., Fujita, Y. \& Katayama, T., Press and Injection Hybrid Molding of Glass Fiber Reinforced Thermoplastics. WIT Transactions on Engineering Sciences, 90(7), pp. 225-232, 2015. 
[11] Tanaka, K., Noguchi, R. \& Katayama, T., Effects of Preheating Temperature on the Interfacial Tensile Strength for Glass Fiber Reinforced Polypropylene Composites Made by Press and Injection Hybrid Molding. WIT Transactions on The Built Environment, 166, pp. 287-296, 2016.

[12] Tanaka, K., Kondo, Y. \& Katayama, T., Effect of Mold Temperature on Interfacial Welded Strength and Outer Shell Laminate Strength of CF/PA6 Composites Manufactured by Press and Injection Hybrid Molding. WIT Transactions on The Built Environment, 166, pp. 317-327, 2016.

[13] Tomari, K., Hamada, H. \& Maekawa, Z., Study on the Weld Strength of Fiber Reinforced Polyamide Injection Moldings. Journal of JSPP, 1(1), pp. 71-78, 1989. 